\title{
Anticoncepcionais hormonais disponíveis no mercado brasileiro
}

Nelson Vitiello*

A maioria dos profissionais que se dedicam à Educação Sexual é constituída de especialistas de outras areas que não a medicina (pedagogia, psicologia, e outras) ou mesmo de médicos não ginecologistas. Para estes profissionais sempre é difícil emitir opiniões ou dar respostas às perguntas das usuárias de métodos anticoncecpcionais hormonais pois, embora possam (e devam) ter conhecimentos sobre os aspectos básicos desse tema, não conhecem a nomenclatura dos produtos comerciais disponíveis em nosso meio. Assim, mesmo quando tenham conhecimentos sobre vantagens e desvantagens de produtos tipo conjugados ou de microdose de progestágenos, dificilmente saberão identificar, pela marca comercial, quais os produtos disponíveis com essas constituições.

Pesquisando entre laboratórios farmacêuticos, farmácias e publicações de bulários, encontra-se um número relativamente grande de anticoncepcionais hormonais. Ocorre, além da diversidade de tipo, repetições

\footnotetext{
* Ginecologista. Doutor em Medicina. Sociedade Brasileira de Estudos em Sexualidade Humana.

Recebido em 01.03.99

Aprovado em 18.03.99
} 
de produtos com as mesmas formulações, fabricados por laboratórios diferentes e com marcas comerciais distintas.

Por tudo isso julgamos que seria de utilidade divulgar, aos profissionais que se ocupam da Educação Sexual, os tipos e marcas comerciais desses produtos disponíveis no mercado brasileiro.

A listagem foi organizada por tipo (oral ou injetável) e, quando cabível, sub-tipo (conjugados ou de microdoses de progestágenos), além das subdivisões existentes dentro de alguns desses sub-tipos (monofásicos, bifásicos, etc.).

A constituição de cada tipo é apresentada em abreviações, que são especificadas no final do texto.

Evidentemente, por se tratar de um mercado dotado de grande dinamismo, constantemente são lançados produtos novos, bem como retirados do mercado alguns dos mais antigos. Assim, a presente listagem reflete a realidade atual do mercado, devendo ser constantemente revista.

Foram encontradas 28 marcas comerciais de anticoncepcionais hormonais conjugados, distribuídas entre 23 monofásicos, 1 bifásico e 4 trifásicos. Foram encontradas também 1 marca de anticoncepcional oral cíclico, 3 marcas de produtos tipo microdose de progestágeno, 5 injetáveis conjugados e 1 de injetável apenas com progestágeno.

No total, considerando-se todo o universo dos anticoncepcionais do tipo hormonal, existem no mercado brasileiro 38 marcas comerciais a disposição das usuárias.

I - Anticoncepcionais hormonais orais conjugados

a) Monofásicos

\begin{tabular}{lcclc}
\multicolumn{1}{c}{ Nome comercial } & No compr. $^{\circ}$ & Subst. ativa & Dose & Placebo \\
\hline 1- Anacyclin & 21 & EE & $50 \mathrm{mcg}$ & - \\
& & LT & $1.000 \mathrm{mcg}$ & - \\
2 - Anfertil & 21 & EE & $50 \mathrm{mcg}$ & - \\
3 - Ciclo 21 & & LN & $150 \mathrm{mcg}$ & - \\
& 21 & EE & $30 \mathrm{mcg}$ & - \\
4 - Ciclovulon & & LN & $150 \mathrm{mcg}$ & - \\
& 21 & EE & $50 \mathrm{mcg}$ & - \\
& & N & $250 \mathrm{mcg}$ & -
\end{tabular}




\begin{tabular}{|c|c|c|c|}
\hline 5-Diminut & 21 & $\begin{array}{l}\mathrm{EF} \\
\mathrm{G}\end{array}$ & $\begin{array}{l}20 \mathrm{mcg} \\
75 \mathrm{mcg}\end{array}$ \\
\hline 6 -Diane & 21 & $\begin{array}{l}\mathrm{EE} \\
\mathrm{AC}\end{array}$ & $\begin{array}{l}35 \mathrm{mcg} \\
2 \mathrm{mg}\end{array}$ \\
\hline 7 - Evanor & 21 & $\begin{array}{l}\mathrm{EE} \\
\mathrm{LN}\end{array}$ & $\begin{array}{l}50 \mathrm{mcg} \\
250 \mathrm{mcg}\end{array}$ \\
\hline 8 - Femiane & 21 & $\begin{array}{l}\mathrm{EE} \\
\mathrm{G}\end{array}$ & $\begin{array}{l}20 \mathrm{mcg} \\
75 \mathrm{mcg}\end{array}$ \\
\hline 9 - Femina & 21 & $\begin{array}{l}\mathrm{EE} \\
\mathrm{DG}\end{array}$ & $\begin{array}{l}20 \mathrm{mcg} \\
150 \mathrm{mcg}\end{array}$ \\
\hline $10-$ Gestinol & 21 & $\begin{array}{l}\mathrm{EE} \\
\mathrm{G}\end{array}$ & $\begin{array}{l}30 \mathrm{mg} \\
75 \mathrm{mg}\end{array}$ \\
\hline 11 - Gynera & 21 & $\begin{array}{l}\mathrm{EE} \\
\mathrm{G}\end{array}$ & $\begin{array}{l}30 \mathrm{mcg} \\
75 \mathrm{mcg}\end{array}$ \\
\hline 12 - Harmonet & 21 & $\begin{array}{l}\mathrm{EE} \\
\mathrm{G}\end{array}$ & $\begin{array}{l}20 \mathrm{mcg} \\
75 \mathrm{mcg}\end{array}$ \\
\hline 13 - Megestran (28) & 21 & $\begin{array}{l}\text { M } \\
\text { MET }\end{array}$ & $\begin{array}{l}100 \mathrm{mcg} \\
500 \mathrm{mcg}\end{array}$ \\
\hline $14-$ Mercilon & $\begin{array}{l}+7 \\
21\end{array}$ & $\begin{array}{l}\overline{E E} \\
\mathrm{DG}\end{array}$ & $\begin{array}{l}- \\
20 \mathrm{mcg} \\
150 \mathrm{mcg}\end{array}$ \\
\hline 15 - Microdiol & 21 & $\begin{array}{l}\mathrm{EE} \\
\mathrm{DG}\end{array}$ & $\begin{array}{l}30 \mathrm{mcg} \\
150 \mathrm{mcg}\end{array}$ \\
\hline 16 - Microvlar & 21 & $\begin{array}{l}\mathrm{EE} \\
\mathrm{LN}\end{array}$ & $\begin{array}{l}30 \mathrm{mcg} \\
150 \mathrm{mcg}\end{array}$ \\
\hline $17-$ Minulet & 21 & $\begin{array}{l}\mathrm{EE} \\
\mathrm{G}\end{array}$ & $\begin{array}{l}30 \mathrm{mcg} \\
75 \mathrm{mcg}\end{array}$ \\
\hline 18 - Neovlar & 21 & $\begin{array}{l}\mathrm{EE} \\
\mathrm{LN}\end{array}$ & $\begin{array}{l}50 \mathrm{mcg} \\
250 \mathrm{mcg}\end{array}$ \\
\hline 19 - Nordete & 21 & $\begin{array}{l}\mathrm{EE} \\
\mathrm{LN}\end{array}$ & $\begin{array}{l}30 \mathrm{mcg} \\
150 \mathrm{mcg}\end{array}$ \\
\hline 20 - Normamor & 21 & $\begin{array}{l}\mathrm{EE} \\
\mathrm{LN}\end{array}$ & $\begin{array}{l}50 \mathrm{mcg} \\
250 \mathrm{mcg}\end{array}$ \\
\hline
\end{tabular}




$\begin{array}{lllll}21-\text { Primera } & 21 & \text { EE } & 20 \mathrm{mcg} & - \\ 22-\text { Primovlar } & 21 & \text { DG } & 150 \mathrm{mcg} & - \\ & & \text { EE } & 50 \mathrm{mcg} & - \\ 23-\text { Selene } & 21 & \text { LN } & 250 \mathrm{mcg} & - \\ & & \text { EE } & 35 \mathrm{mcg} & -\end{array}$

b) Bifásico

\begin{tabular}{lcccc} 
Nome comercial & $\mathbf{N}^{\text {o }}$ compr. & Subst. ativa & Dose & Placebo \\
\hline Gracial (22) & 7 & EE & $40 \mathrm{mcg}$ & - \\
& & DG & $25 \mathrm{mcg}$ & - \\
& +15 & EE & $30 \mathrm{mcg}$ & -
\end{tabular}

c) Trifásicos

\begin{tabular}{|c|c|c|c|c|}
\hline Nome comercial & $\mathbf{N}^{\circ}$ compr. & Subst. ativa & Dose & Placebo \\
\hline \multirow{10}{*}{1 - Levordiol (28) } & \multirow[t]{3}{*}{6} & $\mathrm{EE}$ & $30 \mathrm{mcg}$ & - \\
\hline & & LN & $50 \mathrm{mcg}$ & - \\
\hline & & - & - & B6 $10 \mathrm{mg}$ \\
\hline & \multirow[t]{3}{*}{+5} & $\mathrm{EE}$ & $40 \mathrm{mcg}$ & - \\
\hline & & $\mathrm{LN}$ & $75 \mathrm{mcg}$ & - \\
\hline & & - & - & B6 $10 \mathrm{mg}$ \\
\hline & \multirow[t]{3}{*}{+10} & $\mathrm{EE}$ & $30 \mathrm{mcg}$ & - \\
\hline & & LN & $125 \mathrm{mcg}$ & - \\
\hline & & - & - & B6 $10 \mathrm{mg}$ \\
\hline & +7 & - & - & B6 $10 \mathrm{mg}$ \\
\hline \multirow{6}{*}{2 - Trinordiol (21) } & \multirow[t]{2}{*}{6} & $\mathrm{EE}$ & $30 \mathrm{mcg}$ & - \\
\hline & & $\mathrm{LN}$ & $50 \mathrm{mcg}$ & - \\
\hline & \multirow[t]{2}{*}{+5} & $\mathrm{EE}$ & $40 \mathrm{mcg}$ & - \\
\hline & & $\mathrm{LN}$ & $75 \mathrm{mcg}$ & - \\
\hline & \multirow[t]{2}{*}{+10} & $\mathrm{EE}$ & $30 \mathrm{mcg}$ & - \\
\hline & & LN & $125 \mathrm{mcg}$ & - \\
\hline
\end{tabular}




$\begin{array}{lllll} & 7 & \text { EE } & 35 \mathrm{mcg} & - \\ & & \text { NET } & 500 \mathrm{mcg} & - \\ 3-\text { Trinovum (21) } & +7 & \text { EE } & 35 \mathrm{mcg} & - \\ & & \text { NET } & 750 \mathrm{mcg} & - \\ & +7 & \text { EE } & 35 \mathrm{mcg} & - \\ & & \text { NET } & 1.000 \mathrm{mcg} & - \\ & 6 & \text { EE } & 30 \mathrm{mcg} & - \\ 4-\text { Triquilar (21) } & +5 & \text { LN } & 50 \mathrm{mcg} & - \\ & & \text { EE } & 40 \mathrm{mcg} & - \\ & +10 & \text { LN } & 75 \mathrm{mcg} & - \\ & & \text { EE } & 30 \mathrm{mcg} & - \\ & & \mathrm{LN} & 125 \mathrm{mcg} & -\end{array}$

II - Anticoncepcionais hormonais orais cíclicos

\begin{tabular}{lcclc} 
Nome comercial & $\mathbf{N}^{\circ}$ compr. & Subst. ativa & Dose & Placebo \\
\hline \multirow{2}{*}{ Biofim Trimestral } & Total 28 & & & \\
& & $\mathrm{M}$ & $80 \mathrm{mcg}$ & - \\
& & $\mathrm{M}$ & $80 \mathrm{mcg}$ & - \\
& & $\mathrm{N}$ & $2.000 \mathrm{mcg}$ & - \\
& & - & - & Placebo
\end{tabular}

III - Anticoncepcionais hormonais orais com microdose de progestágenos

\begin{tabular}{lccll} 
Nome comercial & $\mathbf{N}^{0}$ compr. & Subst. ativa & Dose & Placebo \\
\hline 1 - Exluton & 28 & LT & 500 & - \\
2- Micronor & 35 & NET & $350 \mathrm{mcg}$ & - \\
$3-$ Nortrel & 28 & LN & $30 \mathrm{mcg}$ & -
\end{tabular}


IV - Anticoncepcionais hormonais injetáveis conjugados

\begin{tabular}{lll} 
Nome comercial & Subst. ativa/ampola & Dose \\
\hline 1 - Cicrovular & EnE & $10 \mathrm{mg}$ \\
& AA & $150 \mathrm{mg}$ \\
$2-$ Evitas & EnE & $10 \mathrm{mg}$ \\
& AA & $150 \mathrm{mg}$ \\
$3-$ Mesigyna & VE & $5 \mathrm{mg}$ \\
& EN & $50 \mathrm{mg}$ \\
$4-$ Perlutan & EnE & $10 \mathrm{mg}$ \\
& AA & $150 \mathrm{mg}$ \\
$5-$ Unociclo & EnE & $10 \mathrm{mg}$ \\
& AA & $150 \mathrm{mg}$
\end{tabular}
V - Anticoncepcionais hormonais injetáveis só com progesterona

\begin{tabular}{lcc} 
Nome comercial & Subst. ativa/amp. & Dose \\
\hline Depo-Provera & AMP & $150 \mathrm{mg}$
\end{tabular}

\section{LEGENDA}

AA - Acetofenido de Algesterona

AC - Acetato de Ciproteroni

AMP - Acetato de Medroxiprogesterona

DED - Diacetato de Etinodiol

DG - Desogestrel

ED - Etinodiol

EE - Etinil Estradiol

EN - Enantato de Noretisterona
EnE - Enántato de Estradiol

G - Gestodene

LN - Levonorgestrel

LT - Linestrenol

M - Mestranol

$\mathrm{N}$ - Noretindrona

NET - Noretisterona

VE -.- Valerato de Estradiol 